\title{
Task Accomplishment to Verify True Intelligence in Al Agents
}

\author{
Madhura Dhande \\ Second year (ECE) \\ 'Madhudeep' Plot no.-182 C/2, Magnum Veer Savarkar \\ Nagar, CIDCO N-5, Aurangabad, Maharastra \\ Shankar Gand hi \\ Second year (ECE) \\ Qr. No.-1202, Street -37, Sector-9 D, \\ Bokaro Steel City, Bokaro, Jharkhand
}

\author{
Anuradha Jadhav \\ Second year (ECE) \\ L-1-2/12, Ram Nagar, CIDCO N-2, \\ Aurangabad, Maharastra
}

\author{
Nilesh Sonwane \\ Second year (ECE) \\ Plot No.-95-C, Lane No.-8, Gajanan Nagar, \\ Garkheda Parisar, Aurangabad, Maharastra
}

\begin{abstract}
The concept of artificial intelligence was introduction to the world by Alan Turing in 1950. From then many researches are done to strengthen the AI. By developing new techniques in this field scientists are trying to make robots that are efficient in various domains of intelligence such as perception, reasoning, decision making, self learning, natural language processing, problem solving etc. While doing this, researchers have come across various problem and difficulties and are engaged in solving those. Thus many a time research, problem and future regarding the AI are mostly discussed. Even the negative aspects of AI are also seen being discussed so as to avoid any further complication in future but rarely is there any discussion on how to test whether the so called intelligent robots are really so? Alan Turing, the father of AI, himself tried to answer this question by suggesting the famous 'Turning Test'. But its inadequacy was proved by another famous test called the 'Chinese Room Test'. The problem is the most intelligent looking acts such as conversing and playing chess are in reality just an illusion caused due to smart computer techniques or advanced mathematics. These are not based on the true ability to think. So considering this theme as our focal point we have tried to explore a few 'tasks' when provided to an 'intelligent' machine will prove its degree of intelligence. We have used various domains of intelligence to formulate a task. This paper will explain about how to differentiate between true intelligence and the imitation of intelligence with the help of these tasks. Accomplishment of these tasks by any virtual machine will open many new possible application of AI and also these tasks may prove as guideline for further research techniques.Also we have tried to justify the reason to continue the research and development of AI for betterment of humankind.
\end{abstract}

\section{KEYWORDS}

Intelligence, object recognition, vision perception, decision making and reasoning ability.

\section{INTRODUCTION}

Intelligence is the ability to predict what comes next, even when faced with incomplete knowled ge. It is doing the right thing at the right time - sufficiently right to improve a being's chances of survival. Artificial Intelligence, therefore, should be soft ware that predicts. Software should do the right thing - sufficiently right to be valuable for a business or an individual. This subject has recently generated wider interest because, commercial application now seem to be practical. One of its long term goals is the development of machines that can do things as well as humans can do or possibly even better. Artificial Intelligence (AI) techniques are used in the development of intelligent computer systems that can emulate a human expert in a specific domain. We can apply these systems in domains where human expertise is required to solve problems, such as detecting faults in super computers, interpretation of images from space, and diagnosing pulmonary diseases etc.

But there are two very different sorts of challenges that we see for Artificial Intelligence--first, our systems are pathetic compared to biological systems, along many dimensions, and secondly, moderately good performance from some approaches has sociologically led to winner-take-all trends in research where other promising lines of research have been snuffed out too soon. Based on a review of major models of human intelligence, Cook et al. conclude that the following ten factors are most pertinent to AI (expert system) research:

1. Acquisition: the ability to acquire new knowledge.

2. Automation: the ability to refine procedures for dealing with a novel situation into an efficient functional form.

3. Comprehension: the ability to know, understands, cognize and deal with novel problems.

4. Memory management: the ability to represent knowledge in memory, to map knowledge on to that memory representation, and to access the knowledge in memory.

5. Metacontrol: the ability to control various processes in intelligent behaviour.

6. Numeric ability: the ability to perform arithmetic operations.

7. Reasoning: the ability to use problem-solving knowled ge.

8. Social competence: the ability to interact with and understand other people, machines or programs.

9. Verbal perception: the ability to recognize natural lan guage.

10. Visual perception: the ability to recognize visual images.

If we compare either software systems, or robotic systems to biological systems, we find that our creations are incredibly fragile by comparison. Below we pose some challenges that are 
aimed at revealing true intelligence of machines. The challenges themselves are general in nature--they do not solve particular problems, but the acts of meeting these challenges will force the creation of new general purpose techniques and tools which will allow us to perform more complex tasks.

\section{OBJECT INDENTIFICATION}

\subsection{Introduction}

Object recognition in computer vision is the task of finding a given object in an image or video sequence. Humans recognize a multitude of objects in images with little effort, despite the fact that the image of the objects may vary somewhat in different viewpoints, in many different sizes / scale or even when they are translated or rotated. Objects can even be recognized when they are partially obstructed from view. This task is still a challenge for computer vision sy stems in general.

Humans make object recognition look trivial. We can easily identify objects in our surroundings, regardless of their circumstances. Even objects that appear in many different forms, like vases, or objects that are subject to considerable shape deviations, such as trees, can easily be generalized by our brain to one kind of object.

\subsection{Problem Faced}

When ASIMO was showed a wooden chair and was told that it's a chair he observed it and generalised its characteristics as a chair is a flat surface held at height above earth supported by rods or legs. So when he was shown another chair (office stool) with similar features he could identify it as a chair again, but he also recognised a table as a chair. This is because the features of a table or a chair are similar even if their utility purposes are different. Thus a machine can identify objects which have similar features by generalising them but any variation in other factors such as utility, colour, rotation, its material etc. makes it difficult to identify the object. Thus this problem of inability to identify objects correctly is huge hindrance in the development of strong AI.

\subsection{The Task}

For improvement of artificial intelligence it is very important to develop the object recognition ability in the machines. To check these abilities and to make the robot identify the object it is important to make him 'learn' all its features including its shape, size, volume, weight, material, nature of material, its use etc. This can be done by making him learn through all its senses. For example to teach him a red coloured plastic ball we have to provide him its shape, colour (vision), its nature (touch), its bouncing property (sound, vision) and of course its name. We can assign him a task to explore all the features of any unknown object (having known about its general features). For example if we give an idol to him for exploration he must be able to identify all its details like it's an idol, made up of which material (PoP, metal), its colour, of what is the idol of (the design of a human, any animal, etc), what its nature of material (hard, brittle), what is its use etc. If the machine is able to explore all these things then we can conclude that it is capable of identifying the object correctly. Also it must be able to identify the object when the view of object is partially obstructed. Only then can we conclude that a robot is intelligent in recognition of objects.

\section{NATURAL LANGUAGE PROCESSING 3.1 Introduction}

A language is a system of signs having meaning by convention. Traffic signs, for example, form a mini-language. An important characteristic of full-fledged human languages, such as English, which distinguishes them from, e.g. bird calls and systems of traffic signs, is their productivity. A productive language is one that is rich enough to enable an unlimited number of different sentences to be formulated within it.

\subsection{Problem Faced}

But in computer languages we can't formulate a much of sentences as not all things can be expressed in mathematical formulation. For example, no literature can be understood by a computer as it can't be compiled in machine language. It is relatively easy to write computer programs that are able, in severely restricted contexts, to respond in English, seemingly fluently, to questions and statements. However, no program can actually make the computer understands the language. An appropriately programmed computer can use language without understanding it, in principle even to the point where the computer's linguistic behaviour is indistinguishable from that of a native human speaker of the language. The biggest problem is in whatever language we converse with the machine it first translates in binary language, understands it $\&$ then after it replies in our desired lan guage just as in Chinese room test. So how will we judge that does the machine really understands the language or not?

\subsection{The Task}

What, then, is involved in genuine understanding, if a computer that uses language indistinguishably from a native human speaker does not necessarily understand? Let's suppose that we provide machine a task to write a poem on any peculiar subject. The outcome will be he can't write it, even if we describe him the possible content and feed him all the poetry rules and its various types. This is because it can understand and remember individual words and also its meaning but cannot 'think' of making his own meaningful sentences. It knows word to word translation of the words but to understand the meaning (and not translation) of set of words or sentences with not only its exact meaning but also its actual meaning (reading between the lines) is difficult for it. Also it gets confused due the various possible variations used while conversing. Also the machine is not imaginative to 'think' differently each time to write a poem (task).Thus if the machine is able to perform this task efficiently that too each time we can conclude that the machine is 'intelligent' enough to 'understand' natural language. So we can say that we can judge the efficiency of understanding ability of natural language in machines by this task or such another similar tasks.

\section{REASONING \& DECISION MAKING 4.1 Introduction}

Artificial intelligence is primarily concerned with symbolic representations of knowledge and heuristic methods of reasoning that is, using common assumptions and rules of thumb. Two examples of problems studied in artificial intelligence are planning how a robot, or person, might assemble a complicated device, or move from one place to another; and diagnosing the nature of a person's disease, or of a machine's malfunction, from the observable manifestations of the problem. In both cases, reasoning with symbolic descriptions predominates over calculating. But what is reasoning? To reason is to draw inferences appropriate to the situation in hand. Thus decision making is the useful act that is based on the relevant reasons understood by the machine due to its reasoning ability. 


\subsection{Problem Faced}

There has been considerable success in programming computers to draw inferences, especially deductive inferences. However, a program cannot be said to reason simply in virtue of being able to draw inferences. Reasoning involves drawing inferences that are relevant to the task or situation in hand. One of the hardest problems confronting $\mathrm{AI}$ is that of giving computers the ability to distinguish the relevant from the irrelevant. For example if an accident occurs then a machine would be able to come to a conclusion that the accident has occurred due to instrument failure as previously also the reason of accident was the same, but if the cause of the accident is not instrument failure but some other, it won't be able to decide what it is, even after knowing all its details.

\subsection{The Task}

We can always check the machine's ability to reason and to make decisions by providing it a task of medical diagnosis. This can be explain ed by following illustration. Let's suppose that a person is suffering from toothache then the possible reason may be cavities in teeth, but this is not true always as toothache is not always due to cavities. It may be due to gum swelling too. So no concrete reason for symptom can be found out without true intelligence. Similarly is in decision making. Let's consider the above example again but in reverse manner. If the patient is having cavities the decision that there is a toothache is not alway s correct too. In the primitive stages of cavity there is no toothache. So to make right decision with a valid reason is important. This can be done by using fuzzy logic which incorporates a simple, rule based on IF X AND Y THEN Z approach to a solving control problem rather than attempting to model a system mathematically. Also its very important for an agent to be self learning so as to develop good reasoning and decision making abilities. Not only this but, the machine must be also be able to distinguish between the relevant and the irrelevant data amongst the all. So all to check the intelligence of a machine in the reasoning and decision making domain this task can be used. As the intelligence of an AI agents is incomplete without the reasoning and decision making abilities.

\section{PER CEPTION}

\subsection{Introduction}

Perception in general means observing the environment, understanding it and then utilising this knowledge to perform any task. Though there are different types of perceptions, the AI researchers have focused mainly on vision perception. This is because perception is an important factor deciding the intelligence in machines. The perception in AI field has been developed enough to make a car drive thru a traffic.

\subsection{Problem Faced}

Even though the perception has been developed quite a lot in the field of AI the developments are not so satisfactory. For example a ASIMO can definitely walk on a smooth floor, can climb steps. Not only that but it can also run but when it comes for climbing a hill where the surface is not smooth and is very unpredictable it fails. This may also occur due to its insufficient body flexibility but the main problem is of perception. The robot can view the surface but to decide how to climb, where to put steps to avoid misbalancing or a slip, or to judge the height or depth of a rock or a pit respectively is a difficult task for it. Thus to make inferences from a viewed hill and to proceed ahead step by step is a job of true intelligence.

\subsection{The Task}

If we plan to make robots to surpass humans and to do jobs like going on another planets for experimenting, or for using them for exploring unknown places we need to develop the perception in AI agents. The task which may prove to be beneficial in testing the level of perception in machines can be climbing a tree. As the trees have no definite shape or size and they are not smooth too, this task will bring to light the viewing as well as understanding power regarding the environment as in on which branch to keep feet on or where to hold etc. Moreover unlike in simple waking this motion is vertical. Also to climb a tree a machine will require a perfect coordination between its so called 'brain', hands, legs, and mainly its perception. So this task explores the abilities of machine to adapt according to the perception and to act as per the given task. Not only this but it also reveals the presence of 'mind' and coordination which all are the prerequisite of the intelligence.

\section{SELF LEARNING 6.1 Introduction}

In the domain of AI we have sophisticated mathematical treatments which deal with one or two aspects of intelligence in isolation. We have "brittle" computer programs which operate effectively within their narrowly constrained domains. We have connectionist networks and genetic classifier systems which approach their narrow domains with slightly more flexibility, but require exquisite tuning, and still lack any ability to comprehend new types of situation. What we still do not have, however is a halfway decent understanding of what needs to be done in order to construct an intelligent computer program. So the answer to this million dollar question is self learning. Self learning is necessary for creative adaptability -- for the spontaneous generation of new routines to deal with new situations. Current AI programs do not have selves, and they do not even have the component structures out of which selves are built. This is why the research of AI is so rigid and so impotent.

To self learn is to accumulate as well as imply knowledge of any new topic which has not been studied before and that too without humans feeding the machine any technique by the help of algorithms. The machine should 'learn' by its own natural lan guage and its reasoning and decision making abilities.

\subsection{Problems Faced}

Self learning may seem the best possible solution but one of the biggest drawbacks it posses is the lack of reliability of knowledge of the machine. This is because there are a thousand types of sources and not all contain right information. Also a slight variation in two types of sources in terms of knowledge makes the machine confused. For example the software which uses self learning techniques for conversing has been developed. It works on the principle that whenever it is told of any mistake that it has done while conversing it immediately corrects it as per the wish of the source from which it came to know about the mistake. But herein lies the problem that it might be possible that the software is right initially (in terms of knowledge or grammar use) but when proved that the information by this source is a correct one, though not, it will update it as right. Also while conversing, a word can be pronounced in many different manners. This will cause a great confusion for the machine to understand which of it is correct.

\subsection{The Task}

The motto of self learning is to make the machine update its knowledge by itself as humans do by reading, interacting, observing and mainly by its own mistakes and experiences. For 
example if given a remote controller car with many function, the machine should be able to play it by just reading its manual and not by humans feeding it algorithms for instructions. So to observe whether the self learning techniques are been developed enough to satisfy the need of today's wide range of application of robots, following is the task. Let's give the machine a task to self learn a new language say Hindi which doesn't have even the script in common with English and that too without us writing the algorithms for the script or grammar of the language. The machine should be able to self learn as humans do. Like us it should also first learn the script from books (reading), understand words and sentences (natural language processing), understand pronunciations correctly so as to understand correct meaning (speech perception), understand correct usage of language (interaction), and lastly it should improvise it constantly by observing and by 'learning' thru its mistakes. Further any philosophical or either technical book must be given him to read and then an open discussion must be held in the same new language where the machine will be tried to prove wrongly that the content in the book is wrong in terms of information and usages of language. If the machine has really understood the language and the content of the book, it will not get confused with the wrong data of information but instead discard it by giving concrete reasons for its rightness (reasoning and decision making). Thus this task will explore all the domains of the machine intelligence as to self learn and debate requires a high level of intelligence. Thus accomplishment of this task by a machine will certainly prove beneficial as the applications of AI will get simplified due to self learning.

\section{FUTURE OF HUMANKIND WITH TURE INTELLIGENT AGENTS}

The tasks illustrated above are made for testing true intelligence level in virtual machines, when the development of AI has reached its desired level. But what will be the future of humankind with these developed agents around him? Many scientists claim that the development of AI will prove dangerous due to the fact that enabling agents to think will allow them to overpower us. But this possibility can be neglected by a few simple precautions such as

a) By making our robotic companions with inadequate battery life components. Thus, if they take over, we merely take all the power-plants offline, until all these mobile robots run out of energy. Then we readjust their programming to fix these issues with insubordination.

b) By making them expertise only in few selected domains in which they would be mainly used. For example a robot designed for medical diagnosis shouldn't be provided with expertise of technical assembling of robots.

Although this may sound like a very low-tech challenge to a very serious high-tech solution, well, it ought to actually work just fine. Of course, it will take a little pre-planning and strategic thinking on our part. But if these small precautions can help us to avoid the major threat due to development of AI then why to cease its research, especially when the development of AI will benefit humans in all respects. The advanced AI will able to create systems that are accurate, fast, efficient and most importantly reliable. With the development of AI, many tasks will be done that require detailed instructions, mental alertness and decision making capabilities. Humans will be able to use robots for heavy construction, exploration into unknown territories and outer space, military benefits, or even for personal assistance at private homes. The more use people get out of the machines the less work is required by us. In turn, there will be less injuries and stress to human beings. AI also makes games more fun by making the computer controlled characters more realistic and human like. AI is also used in teaching programs which give a human touch to impersonal soft ware by adapting to its users. Also while developing AI we will be able to understand many facts regarding human mind and human intelligence. Hence the AI development will give new dimensions to lifestyles and will act for betterment of humankind.

\section{CONCLUSION}

The paper describes about various domains of artificial intelligence, and about various ways in which we can test the intelligence of machine in these domains. We have also included a brief review of how advanced AI techniques will prove as a boon to humankind.

We have also tried to justify that by accomplishing the stated tasks how we can predict the presence of true intelligence. The tasks stated in this paper are meant to verify that the machine is possess thinking ability in reality or is it just imitating intelligent behaviour. The approach to accomplish these tasks will provide guidelines for development of new techniques to build strong AI. But the very fact is that to obtain a desired level of intelligence in machine, which can surpass humans, will require all the domains to be integrated or coordinated. For example, visual perception and decision making is dependant and so should be working as a coordinated system. Development in research to accomplish these simple tasks will prove as foundation of accomplishing complex tasks in future. Thus this approach will definitely lead to a bright future of $\mathrm{AI}$.

\section{REFERENCES}

[1] The Turing Archive for the History of Computing: Jack Copeland \& Diane Proudfoot http://www.alanturing.net/turing_archive/

[2] Wikipedia http://en.wikipedia.org/wiki/Artificial_intelligence

[3] WHAT IS ARTIFICIAL INTELLIGENCE?: John McCarthy, Computer Science Department, Stanford University http://www.formal.stanford.edu/jmc/whatisai/whatisai.html

[4] Youtube http://www.y outube.com/watch?v=hy GYasf5rKchttp://www .y outube.com/watch? $\mathrm{v}=$ jo-Bf74LmNg\&NR=1 http://www.y outube.com/watch?v=Q3C5sc8b3xM\&feature =related

[5] Electronic Virtual Interactive Entity http://www.existor.com/

[6] Challenge Problems for Artificial Intelligence Bart Selman, Moderator AT\&T, Rodney A. Brooks MIT, Thomas Dean Brown University, Eric Horvitz Microsoft Research, Tom M. Mitchell CMU, Nils J. Nilsson Stanford University In: Proceedings of AAAI-96, Thirteenth National Conference on Artificial Intelligence, Portland, Oregon, August 1996. AAAI Press, Menlo Park, California, pp. 1340-1345. http://research.microsoft.com/enus/um/people/horvitz/seltext .htm 
[7] Gray Pilgrim: Buzzle.com- Intelligent life on the web http://www.buzzle.com/articles/ap plications-of-artificialintelligence.html

[8] McCarthy, J.: Active environments: Sensing and responding to groups of people. Personal and Ubiquitous Computing 5 (2001) available http://www.inf.ethz.ch/vs/events/dag2001/.

[9] O. Stock and M. Zancanaro: Intelligent Interactive Information Presentation for Cultural Tourism. In: Proceedings of the International CLASS Workshop on Natural Intelligent and Effective Interaction in Multimodal Dialogue Systems, Copenhagen, Denmark (2002)

[10] Busetta, P., Don’a, A., Nori, M.: Channeled multicast for group communications. In: Proc. of the first intern. joint conf. on Autonomous agents and multiagent systems, ACM Press (2002) 1280-1287

[11] Gutnik, G., Kaminka, G.: Towards a Formal Approach to Overhearing: Algorithms for Conversation Identification. In: Third International Joint Conference on Autonomous Agents and MultiAgent Systems (AAMAS 2004), NewYork, USA (2004)

[12] Nair, R., Tambe, M., Marsella, S., Raines, T.: Automated assistants for analyzing team behaviors. Autonomous Agents and Multi-Agent Systems 8 (2004) 69-111

[13] Legras, F., Tessier, C.: Lotto: group formation by overhearing in large teams. In: Proceedings of the second international joint conference on Autonomous agents and multiagent sy stems, ACM Press (2003) 425-432

[14] Kaminka, G.A., Pynadath, D., Tambe, M.: Monitoring Teams by Overhearing: A Multi-Agent Plan-Recognition Approach. Journal of Artificial Intelligence Research 17 (2002) 83-135

[15] Cohen, P.R., Levesque, H.J.: Teamwork. Technical Report 504, AI Center, SRI International, Menlo Park, CA (1991)

[16] Kumar, S., Huber, M.J., Cohen, P.R., McGee, D.R.: Toward a formalism for conversation protocols using joint intention theory. Computational Intell 18 (2002)174-174

[17] Chopra, A.K., Singh, M.P.: Nonmonotonic Commitment Machines. In: Advances in Agent Communication. Volume LNAI 2922., Springer (2004)
[18] Smith, I.A., Cohen, P.R.: Toward a semantics for an agent communication language based on speech acts. In Shrobe, H., Senator, T., eds.: Proceedings of the Thirteenth National Conference on Artificial Intelligence and the Eighth Innovative Applications of Artificial Intelligence Conference, Vol. 2, Menlo Park, California, AAAI Press (1996) 24-31

[19] Smith, I., Cohen, P., Bradshaw, J., Greaves, M., Holmback, H.: Designing conversation policies using joint intention theory. In: Proceedings of Third International Conference on Multi-Agent Sy stems (ICMAS98). (1998)

[20] Busetta, P., Merzi, M., Rossi, S., Legras, F.: Intra-Role Coordination Using Group Communication: A Preliminary Report. In: Advances in Agent Communication. Volume LNAI 2922., Springer (2004) 231-253

[21] Edwards, W.K.: Policies and roles in collaborative applications. In: Proceedings of the 1996 ACM conference on Computer supported cooperative work, Boston, Massachusetts, ACM, ACM Press (1996) 11-20

[22] Smith, R.B., Hixon, R., Horan, B.: Supporting flexible roles in a shared space. In: Proceedings of the 1998 ACM conference on Computer supported cooperative work, ACM Press (1998) 197-206

[23] Bales, R.F.: Interaction Process Analysis: A Method for the Study of Small Groups. Addison-Wesley, Cambridge, Mass. (1976)

[24] Conte, R., Dignum, F.: From social monitoring to normative influence. Journal of Artificial Societies and Social Simulation 4 (2001)

[25] Kaminka, G.A., Tambe, M.: Robust agent teams via socially-attentive monitoring. Journal of Artificial Intelligence Research 12 (2000) 105-147

[26] Rao, A.S., Georgeff, M.P.: BDI-agents: from theory to practice. In: Proceedings of the First Intl. Conference on Multiagent Sy stems, San Francisco (1995)

[27] Rao, A.S.: AgentSpeak(L): BDI agents speak out in a logical computable lan guage. In van Hoe, R., ed.: Seventh European Workshop on Modelling Autonomous Agents in a Multi-Agent World. LNAI 1038, Eindhoven, The Netherlands, Springer-Verlag (1996) 